\title{
The Diffuse Interstellar Bands: an Elderly Astro-Puzzle Rejuvenated
}

\author{
Nick L. J. Cox ${ }^{1}$ \\ ${ }^{1}$ Instituut voor Sterrenkunde, K.U.Leuven, \\ B-3000, Leuven, Belgium \\ email: nick.cox@ster.kuleuven. be
}

\begin{abstract}
The interstellar medium constitutes a physically and chemically complex component of galaxies and is important in the cycle of matter and the evolution of stars. From various spectroscopic clues we now know that the interstellar medium is rich in organic compounds. However, identifying the exact nature of all these components remains a challenge. In particular the identification of the so-called diffuse band carriers has been alluding astronomers for almost a century.

In recent decades, observational, experimental and theoretical advances have rapidly lead to renewed interest in the diffuse interstellar bands (DIBs). This has been instigated partly by their perceived relation to the infrared aromatic emission bands, the UV extinction bump and far-UV rise, and the growing number of (small) organic molecules identified in space.

This chapter gives an overview of the observational properties and behaviour of the DIBs, and their presence throughout the Universe. I will highlight recent progress in identifying their carriers and discuss their potential as tracers and probes of (extra)-Galactic ISM conditions.
\end{abstract}

Keywords. astrochemistry, line: identification, line: profiles, polarization, ISM: abundances, ISM: atoms, ISM: clouds, (ISM:) dust, extinction, ISM: lines and bands, ISM: molecules

\section{Introduction}

In this contribution I will review and discuss the properties, behaviour, presence and absence of diffuse interstellar bands (DIBs) in space. To set the stage I will first discuss the diffuse interstellar medium and its chemical complexity (Section 2). Next I will discuss in detail the general properties of the DIBs (Section 3) and their behaviour (Section 4). Section 5 reviews recent work on the presence of DIBs in extra-galactic environments, while Section 6 focuses on observations of the carriers in other astrophysical environments. In the final part (Section 7) recent advances in laboratory astrophysics are highlighted and suggestions for further work and criteria for conclusive identification of carriers are proposed.

\section{The diffuse ISM and its organic constituents}

Diffuse to translucent interstellar clouds have typical temperatures in the range of 50 to $200 \mathrm{~K}$, and particle volume densities between 100 to $1000 \mathrm{~cm}^{-3}$. These colder clumps are immersed and dispersed throughout the low density, warm medium (e.g. Snow \& McCall (2008), Ferrière (2001)). Visual extinctions for individual diffuse clouds are generally less than a few magnitudes. However, the total line-of-sight extinction can be much larger, in particular in the Galactic plane and towards the Galactic Centre. DIBs have been detected in sightlines with visual extinction of $\sim 10$ mag (e.g. Cox et al. (2005)). For (individual) diffuse clouds the chemical networks are driven by photochemistry. The interstellar radiation field (ISRF) penetrates a significant fraction of the cloud's interior, 
even for translucent clouds $\left(A_{V}>4 \mathrm{mag}\right.$; Snow \& McCall (2008)). Nearby stars with strong UV flux can drastically increase the effective UV radiation. The (diffuse) ISM is rich in complex (organic) matter as evidenced from the various identified and unidentified spectral features (Kwok \& Sandford (2008)).

Identified features

Already in the early 1900s a number of atoms, such as Na I, Ca II, Ti II were identified in reddened optical spectra of early type (OB) stars. The first molecules in space were detected through their UV-optical transitions, e.g. $\mathrm{CH}, \mathrm{CH}^{+}$, and $\mathrm{CN}$.. Other molecules were later identified in the $\mathrm{UV}$ (e.g. $\mathrm{C}, \mathrm{CO}, \mathrm{H}_{2}$ ) and the near-infrared (e.g. $\mathrm{C}_{2}, \mathrm{H}_{2}$, $\mathrm{H}_{3}^{+}$). Polyatomic molecules, such as $\mathrm{C}_{3}$ have been detected in the optical (Roueff et al. (2002), Krełowski et al. (2011)) as well as in the far-infrared/sub-mm (Cernicharo et al. (2000), Mookerjea et al. (2011)).

\section{Unidentified features}

Notwithstanding major progress in observational and laboratory astrophysics, several components of the (diffuse) ISM remain unknown. Their spectral signatures suggest primarily carbonaceous "organic" components. In Table 1 we give a brief overview of the unidentified spectral features detected in the ISM. Together the UIB, DIB, UV bump, and $3.4 \mu \mathrm{m}$ feature constitute a significant part $(>30 \%)$ of the available cosmic carbon budget.

Table 1. Unidentified features in the ISM and a non-exhaustive summary of proposed carriers.

\begin{tabular}{|c|c|c|c|c|}
\hline Feature & | Wavelength & $f_{X}$ & | Proposed carriers & $\mid$ ref $\mid$ \\
\hline UV bump & $2175 \AA$ & $\sim 5 \cdot 10^{-5}$ & $\begin{array}{l}\text { graphitic material, fullerenes, } \\
\text { bucky onions, PAHs }\end{array}$ & 1 \\
\hline Diffuse Interstellar Bands & $4060-13175 \AA$ & $\sim 10^{-7}$ & $\begin{array}{l}\text { PAHs, fullerenes, linear carbon } \\
\text { chains }\end{array}$ & 2 \\
\hline 3.4 & 3.4 & & UV processed HACs & 3 \\
\hline Infrared Emission Bands & $3.3,6.2,7.7,8.6,11.3 \mu \mathrm{m}$ & $\sim 10^{-6}$ & PAHs, fullerenes & 4 \\
\hline
\end{tabular}

Notes: The fraction of abundance is defined as $N_{X} / N_{H}$. The uncertainty in $f_{\mathrm{D} \text { IB }}$ is as much as several orders of magnitude (Sect.3).

References: (1) Stecher (1965), Bless \& Savage (1972), Menella et al. (1998), Iglesias-Groth (2004), IglesiasGroth (2007), Mulas et al. (2011); (2) Heger (1922), Merrill \& Wilson (1938), Herbig (1995), Sarre et al. (2006) (and references therein); (3) Butchart et al. (1986), McFadzean et al. (1989), Adamson et al. (1990), Pendleton et al. (1994), Sandford et al. (1995), Chiar \& Pendleton, 2008; (4) Tielens (2011), Peeters, (2011), Cami et al. (2011), Draine (2011), Peeters, (2011).

\section{The diffuse interstellar bands: from first discovery to extensive surveys}

In the early 1900s several observers (e.g. Canon, Heger) noted broad "diffuse" absorption bands on photographic plates of spectra taken of bright stars. Heger (1922) first noted their stationary nature as observed towards a spectroscopic binary, indicating like the known strong stationary sodium and calcium lines - their origin was not stellar, but rather interstellar. The seminal paper by Merrill \& Wilson (1938) presented one of the first in depth systematic studies of the stronger diffuse bands and their relation to both the gas and dust in the ISM. From the broadness of the lines it was immediately evident that these species could not be atomic nor di-atomic in nature. Both atoms and diatomic molecules were detected in space by that time and their intrinsic line widths are much narrower (only a few $\mathrm{km} / \mathrm{s}$ ). Ever since Merrill's work, researchers have tried to answer the inevitable question raised in that paper: 
"What are the carriers of the diffuse - interstellar - bands? And are they due to grains or molecules?"

As of today, more than 400 DIBs have been confirmed towards a few individual linesof-sight probing the diffuse ISM (Hobbs et al. (2008), Hobbs et al. (2009)). The strongest DIBs are detected in no more than a few hundred Galactic and extra-Galactic sightlines. All currently known DIBs lie between 4000 and $10000 \AA$, with two interstellar bands at 1.13 and $1.31 \mu \mathrm{m}$ (Joblin et al. (1990), Rawlings et al. (2011)). A brief summary of the DIB observational properties is given in Table 2. The importance of the DIBs as a chemical component of the ISM is illustrated by the estimated fraction their carriers could constitute with respect the total abundance of PAHs in the ISM per unit reddening (Table 1$): N_{\mathrm{DIB}} \approx 10^{14} \mathrm{~cm}^{-2} \approx 2 \cdot 10^{-8} N_{H} \approx 0.1 N_{\mathrm{PAH}}$ (with $N_{\mathrm{PAH}} / N_{H} \sim 3 \cdot 10^{-7}$ Tielens $(2008)$ and $\left.N_{H} / \mathrm{E}(\mathrm{B}-\mathrm{V})=5 \cdot 10^{21} \mathrm{~cm}^{-2} / \mathrm{mag}\right)$.

Table 2. Key properties of the diffuse interstellar bands

\begin{tabular}{|l|l|}
\hline Property $^{1}$ & Typical value \\
\hline FWHM & $0.5-30 \AA / 2-80 \mathrm{~cm}^{-1} / 50-1000 \mathrm{~km} / \mathrm{s}$ \\
Wavelength & $4000-10000 \AA$ \\
DIB strength - equivalent width & $5-2000 \mathrm{~m} \AA$ per E $(\mathrm{B}-\mathrm{V})$ \\
DIB strength - central depth & up to $20 \%$ per E $(\mathrm{B}-\mathrm{V})$ \\
Peak of number density distribution & $6500 \AA$ \\
Peak of FWHM distribution & $0.7 \AA$ \\
Total equivalent widths & \\
Total abundance N(DIB $)^{3}$ & $\sim 25-30 \AA$ per E $(\mathrm{B}-\mathrm{V})$ \\
\hline
\end{tabular}

Notes:

(1) Global properties gathered from surveys by Jenniskens \& Desert (1994), Herbig (1995), Tuairisg et al. (2000), Hobbs et al. (2008); (2) This is an estimate since the DIB strength per unit reddening vary between lines-of-sight due to changing local environmental conditions; (3) This is a zeroth order estimate assuming a typical oscillator strength, $f$, of unity and transition wavelength of $6500 \AA$ for all DIBs. Small PAHs generally have small $f$ values between 0.01 and 0.1 , thus requiring a higher abundance to give rise to bands of given strength (see Sect. 7).

\section{Environmental behaviour of DIBs}

\section{Dust \& gas}

The equivalent width of diffuse interstellar bands shows a positive trend (albeit with only a weak to intermediate correlation) with the amount of dust (measured via reddening $\mathrm{E}(\mathrm{B}-\mathrm{V}))$ in the line-of-sight $\dagger$ as well as with the amount of gas in the sightline (measured via absorption lines). In Figure 1 we show the relation of the $5780 \AA$ DIB with dust and of the $6614 \AA$ DIB with gas $(\mathrm{N}(\mathrm{K} \mathrm{I})$ ). Similar trends between DIB strength and reddening are observed for many other DIBs (see e.g. Friedman et al. (2011), Vos et al. (2011)). Also, most DIB strengths show a correlation with molecular tracers, such as $\mathrm{CH}$ and $\mathrm{CH}^{+}$, which also correlate roughly with $\mathrm{E}(\mathrm{B}-\mathrm{V})$ (Krełowski et al. (1999), Vos et al. (2011)). A few DIBs show a correlation with $C_{2}$ abundance (Thorburn et al. (2003)).

Although most DIBs are stronger in sightlines with more dust, their relative ratios can differ significantly, by as much as an order of magnitude for sightlines with similar E(B-V). A notable example, is the ratio between the 5780 and 5797 DIB (Krełowski \& Westerlund (1988)). Krełowski \& Sneden (1994) found that, for sightlines with similar reddening, the 5797 band is deeper than the 5780 band if the line-of-sight has as high molecular content ( $\zeta$-type), while it is shallower for sightlines with a low abundance of molecules

$\dagger$ This is partly a selection bias as many DIBs have been confirmed via their increase in strength with $\mathrm{E}(\mathrm{B}-\mathrm{V})$. 
( $\sigma$-type). Further observational studies and models show that the effective strength of the interstellar radiation field (ISRF) is very important in setting the balance between the formation/destruction of molecules as well as their hydrogenation and ionisation state (Le Page et al. (2003), Ruiterkamp et al. (2005), Cox \& Spaans (2006)). Figure 2 shows a schematic drawing of the ISRF affecting diffuse clouds.
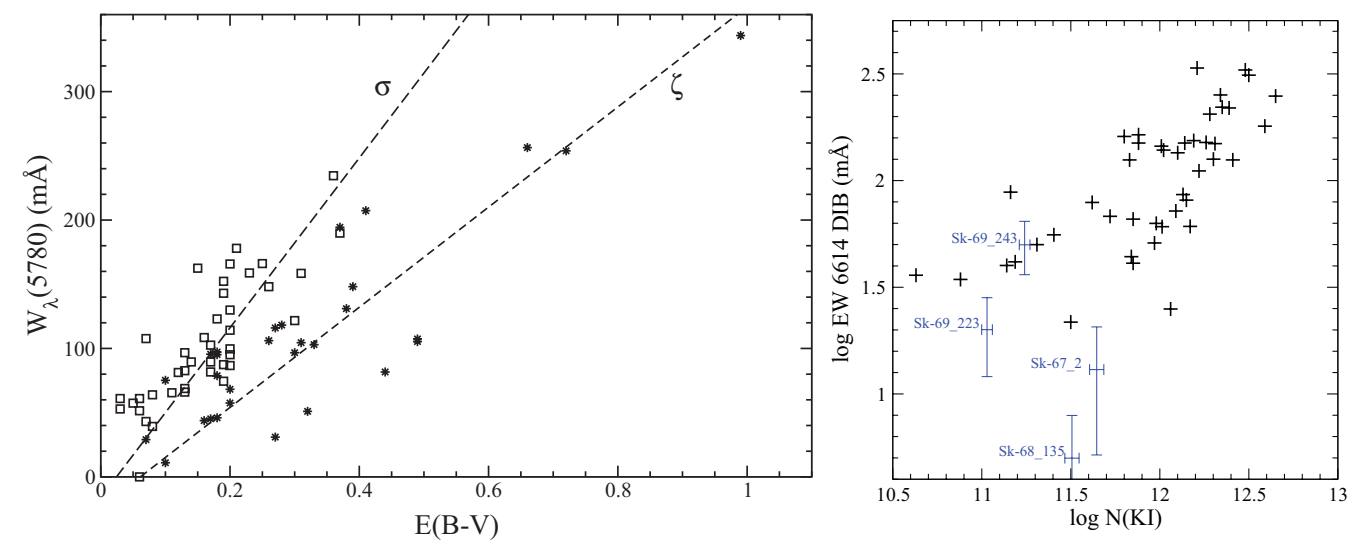

Figure 1. DIB strength versus dust and gas abundance. Left: The $5780 \AA$ DIB equivalent width for Galactic sightlines is plotted as a function of reddening (adapted from Vos et al. 2011). Depending on the ratio between 5780 and 5797 DIBs there is a difference in the slopes of the linear relation for the two sets (e.g. $\sigma$ versus $\zeta$ ). Right: The $6613 \AA$ DIB equivalent width is plotted as a function of $\mathrm{N}(\mathrm{KI})$ (adapted from Cox et al. 2006). Crosses (+) are for Galactic sightlines. Four LMC sources are indicated with error bars. The log-log plots shows a positive trend, although there is large scatter at the lower abundances.

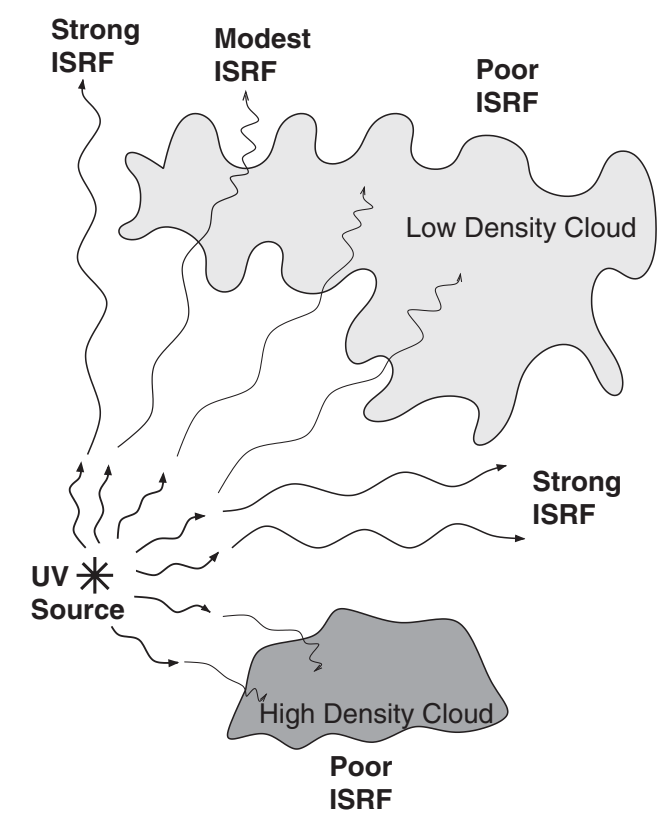

Figure 2. The relative strengths of several diffuse bands are affected by the effective interstellar radiation field (ISRF) impacting the respective interstellar clouds. The schematic drawing illustrates that different types of cloud are exposed to different levels of UV radiation. The low density - UV exposed - cloud is of $\sigma$-type, and the high-density - UV protected - cloud is of $\zeta$-type. Figure courtesy of D. Vos. 


\section{Line polarisation}

Interstellar grains are known to align due to the interstellar magnetic field. Thus carriers directly associated - or even attached to grains - could potentially align as well and thus give rise to a polarisation feature across the profile (Martin \& Angel (1974)). Despite several studies of DIBs in sightlines with strong linear continuum polarisation no DIB polarisation features have been found. The most extensive surveys to date exclude the presence of line polarisation in 40 DIBs to a level of less than $0.01 \%$ (Cox et al. (2007), Cox et al. (2011)). Thus, DIB carriers (at least the ones studied) seem not to be related to large grains nor grain impurities.

\section{The near-infrared DIB at $8621 \AA$}

In particular, the $8621 \AA$ DIB also does not reveal a polarisation signal. This DIB is unique in that it is reported to have a unusually tight correlation with reddening (Wallerstein et al. (2007), Munari et al. (2008)) although independent data show this is not so much the case (Krelowski - see Q\&A section). Also, as it will be observed with the radial velocity spectrograph on the GAIA astrometry mission this DIB will be measured towards thousands of stars in the Milky Way, providing an independent reddening/extinction estimator to de-redden stellar SEDs and/or to construct a 3-dimensional Galactic dust extinction map. Also, this will confirm (hopefully) beyond doubt the ubiquitous presence of DIB carriers throughout the Galaxy (currently DIBs have been detected in only a few hundred lines-of-sight towards Galactic (field) stars).

\section{Substructure in DIB profiles}

High-resolution observations show that many of the narrow - both strong and weak - DIBs reveal sub-structure in their absorption profiles, whereas the broader DIBs have a smooth profile (Sarre et al. (1995), Foing \& Ehrenfreund (1996), Kerr et al. (1996), Galazutdinov et al. (2003)). The observed double or triple peak sub-structures are typical of rotational contours of large (20 to 40 carbon atoms) gas-phase molecules (Danks \& Lambert (1976)). The profiles do not vary in time, but do change with temperature of the ISM (Cami et al. (2004)).

\section{DIB-DIB correlations}

Since most DIBs grow stronger with increasing reddening or gas column density, it is not surprising there is a correlation between individual DIBs. However, it is surprising that almost none of the individual DIBs correlate perfectly with each other. This lack of correlation suggests that the DIBs do not share exactly the same carrier. This is the $1 D I B$ - 1 PAH hypothesis. Recently, the 6196-6613 $\AA$ DIB pair has been reported to show a tight correlation, with a Pearson correlation coefficient of 0.986 (McCall et al. (2010)). This is indeed much better than the rather good correlation between the 5780 DIB and $\mathrm{E}(\mathrm{B}-\mathrm{V})$ or $\mathrm{N}(\mathrm{HI})(\mathrm{r}=0.82$ and $\mathrm{r}=0.95$, resp. Friedman et al. $(2011))$. This tight relation could hint at a common carrier. However, some problems remain. Firstly, the two DIBs have different line widths, shapes and strengths. Secondly, there remains significant scatter on the relation - in particular at low reddening. This puts, at best, strong constraints on the molecular constants of the carrier.

\section{DIBs and the UV bump}

The correlation between DIBs and the UV bump has also been of considerable interest as both features a believed to arise from the organic reservoir of interstellar matter. However, following up on earlier results by Megier et al. (2005)) who compared the farUV rise with DIBs, a recent study by Xiang et al. (2011) shows no significant correlation between the UV bump or far-UV rise and DIB strengths. 
Table 3. Global properties of the Local Group galaxies. See e.g. Cox et al. (2006), Cox et al. (2007), Cordiner et al. (2008a), Cordiner et al. (2008b), Cordiner et al. (2011) and references therein. Note that the gas-to-dust ratio is inversely proportional to the metalicity.

\begin{tabular}{|l|l|l|l|}
\hline Galaxy & Metallicty & ISRF & UV extinction curve \\
\hline Milky Way (MW) & $\sim 1$ & $1-10$ & Nominal. Large scatter on far-UV rise \\
Large Magellanic Cloud (LMC) & $\sim 0.2-0.5$ & $>5$ & Weak far-UV rise, strong ISRF \\
Small Magellanic Cloud (SMC) & $\sim 0.1-0.2$ & $>30$ & No UV bump; strong far-UV rise \\
Andromeda (M31) & $\sim 1$ & $<1$ & Weak UV bump, anomalous extinction curve \\
Triangulum (M33) & $\sim 1$ & $\sim 1$ & \\
\hline
\end{tabular}

\section{Ubiquitous presence of DIBs throughout Galactic and extra-Galactic ISM}

In this section I will report on the presence of DIBs in extra-galactic environments. The study of DIBs throughout time and space is important to understand both the chemical evolution of Galaxies as well as to understand the properties of the carriers themselves. Observations of DIBs can potentially be used as proxies of local environmental conditions of the ISM in distant galaxies.

\section{The Local Group}

The Local Group galaxies offer a unique laboratory to study the behaviour of the DIBs in a wider range of physical conditions than possible within the Milky Way alone (Table 3). In particular the effects of variations in the gas-to-dust ratio, the metalicity, and the interstellar radiation field strength and hardness on the DIBs can be traced.

The LMC, SMC, M31 and M33 are sufficiently nearby (at about 50, 60, 780, and $880 \mathrm{kpc}$, respectively), to discern individual stars and to obtain spectra at high S/N. Nevertheless, $8+\mathrm{m}$ class telescopes are required for high-resolution spectroscopy of stars in the SMC and LMC (Ehrenfreund et al. (2002). For M31 and M33 stars high-resolution spectra are still difficult to obtain, requiring several hours of integration time on the largest telescopes currently available. Low-resolution multi-object spectroscopy using Galactic templates has proven to be very successful (Cox \& Cordiner (2008), Cordiner et al. (2011)). Studies of the Local Group thus offer additional insight into the formation and destruction of DIB carriers.

The LMC and SMC are the nearest Local Group members allowing for detailed studies of, for example, their stellar population and interstellar matter content. DIBs are underabundant in the ISM of the SMC bar, but have normal Galactic strength in the wing (Cox et al. (2006), Welty et al. (2006), Cox \& Cordiner (2008)). The extinction curve for the bar ISM reveals an absence of the UV bump carriers, while the wing ISM does show an UV bump.

M31 and M33 offer unique complete views of spiral galaxies, where we can easily probe different regions in the galaxies, unlike for the Milky Way due to our own vantage point in it. Due to this view from above most M31 and M33 sightlines probe only low to intermediate dust columns, unlike the high extinction lines-of-sight probing the Galactic plane. Although this ensures less confusion due to multiple clouds in a line-of-sight, it also yields intrinsically weaker and thus harder to detect diffuse bands (assuming the Galactic relation holds). A recent survey of DIBs towards 30 sightlines in M31 shows that their strengths show a trend with reddening similar to the one observed for the Milky Way (Cordiner et al. (2011)). The DIBs are on average a little stronger per unit reddening (thus more typical of $\sigma$-type clouds - see Fig. 1), but fall within the envelope of Galactic data points (Figure 3). This effect is likely an observational bias as stronger 

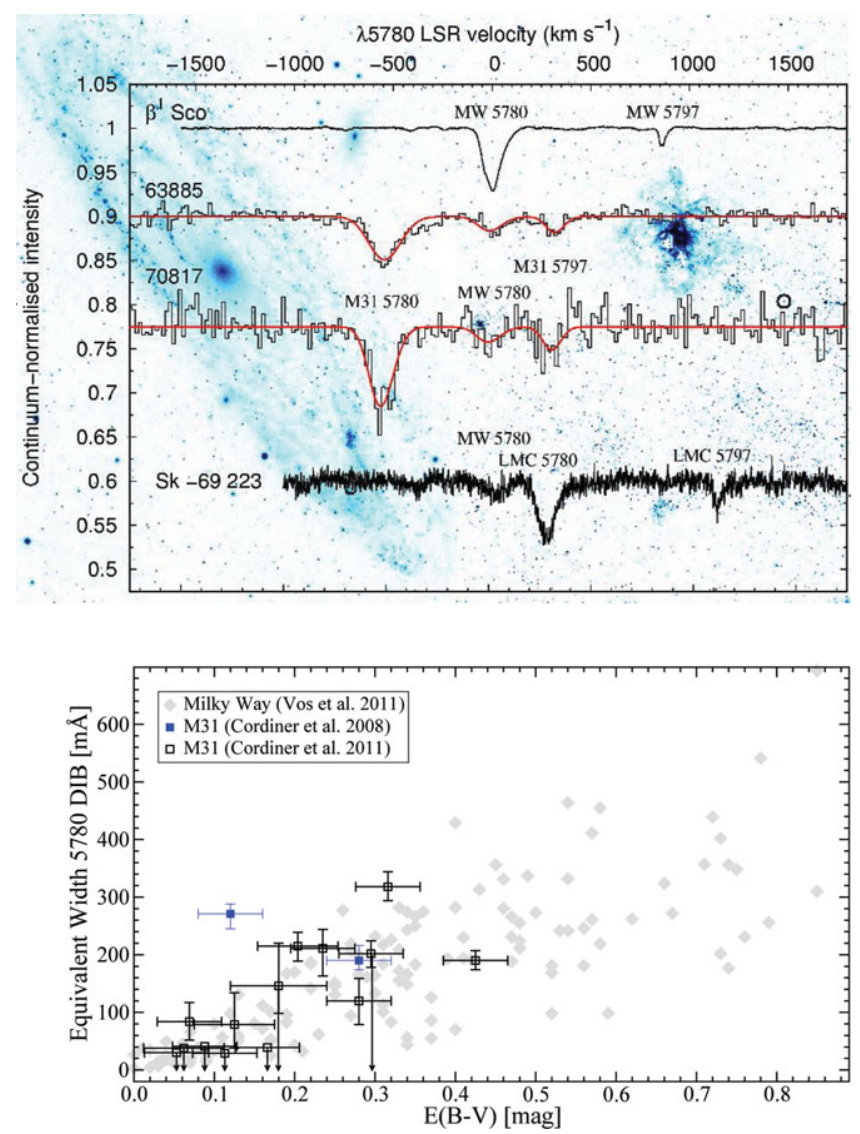

Figure 3. Top: The 5780 and $5797 \AA$ DIBs observed towards the Milky Way, M31 and the LMC. Bottom: $5780 \AA$ DIB equivalent width versus reddening for M31 and the Milky Way lines-of-sight (Adapted from Cordiner et al. 2008, 2011).

lines are more readily detected in low-resolution low-S/N spectra. It is also found that the DIB carrier abundance in M31 does not correlate with either the UV field strength nor the PAH emission. Further high-resolution spectra are needed to probe the physical conditions and radiation field via atomic and molecular lines.

\section{Beyond the Local Group}

Supernovae offer the unique opportunity to study the distant extra-galactic ISM. SNe can become bright enough to act - for a limited time - as background "light houses" suitable for high-resolution spectroscopy of the intervening ISM. Often the study of the ISM is obtained as an added bonus to studied of the temporal evolution of the expanding SNe photosphere. In this way DIBs have been detected in several galaxies such as NGC 1448 (d 17 Mpc; Sollerman et al. (2005)), M100 (d 16 Mpc; Cox \& Patat (2008) and Fig. 4), and NGC 2770 (d 26 Mpc; Thoöne et al. (2009)). The DIB strength ratios observed toward NGC 1448 suggest that the two supernovae, SN2001el and SN2003h, in this galaxy probe a $\sigma$ and $\zeta$ environments, respectively. The line-of-sight towards SN2008D in NGC 2770 is highly extincted with an E(B-V) of 0.8 mag. The measured DIB strengths per unit reddening are similar to the Galactic trend indicating that multiple individual clouds in the ISM of this galaxy are probed. The ISM in M 100 towards SN 2006X is very dense with a high molecular content, and with DIBs per unit reddening more than 7 times weaker than for the Milky Way. 
The 5780 and 6283 DIBs were detected in dusty starburst galaxies. The weaker DIBs at 5797 and 6613 were also detected in the galaxies M 82 and NGC 2146 at 4 and 14 Mpc, respectively (Heckman \& Lehnert (2000)). Despite the harsh conditions expected for the dusty central regions of starburst galaxies the DIBs have similar strengths per unit reddening. Several broad and narrow DIBs have also been detected in the $z=0.52$ Damped Ly $\alpha$ system toward AO 0235+164 DLA (Junkkarinen et al. (2004), York et al. (2006)). Thus, the formation and destruction of DIB carriers depends on the local physical and chemical conditions. And similar conditions exist among different galaxies.

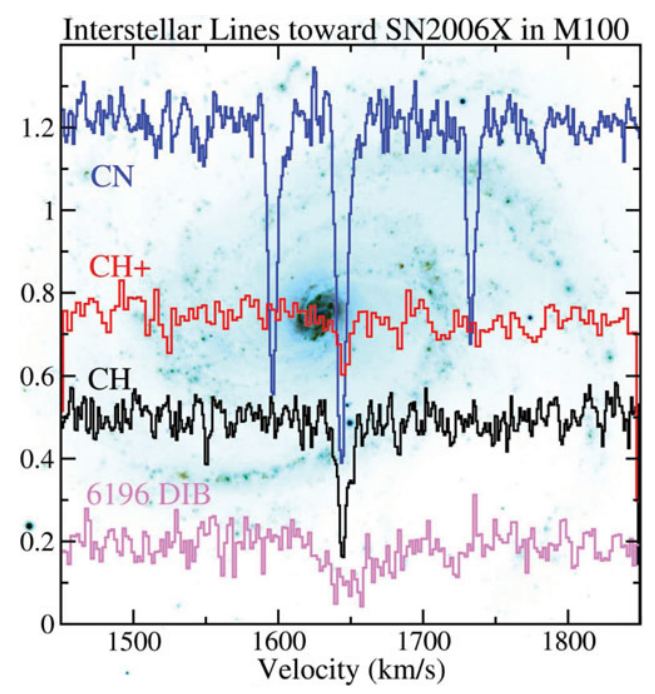

Figure 4. Detection of $\mathrm{CN}, \mathrm{CH}^{+}, \mathrm{CH}$ and $6196 \AA$ DIB in M100 towards SN2006X. M100 is a spiral galaxy seen face-on at a distance of $\sim 16$ Mpc. Adapted from Cox \& Patat (2008). Background image adapted from ESO VLT/FORS.

\section{Diffuse (interstellar) band carriers in other astrophysical environments}

Little is known about the presence of the carriers giving rise to the DIBs in other astrophysical environments. An important question to answer is if DIBs are exclusive to the ISM or that the carriers are present elsewhere? If the DIBs arise from large gas phase molecules such as PAHs and fullerenes it is suggested that they should also be present in other environments rich in organic material. However, diffuse circumstellar bands are absent in dusty circumstellar envelopes of AGB (Mauron \& Kendall (2004)) and post-AGB stars (Luna et al. (2008)), as well as absent in atmospheres of cool stars (Destree et al. (2007)) and Herbig Ae/Be stars (Galazutdinov et al. (2003)). For all these cases the main difficulty is to distinguish the circumstellar diffuse bands from the interstellar diffuse bands. DIBs have been searched for - without success - in comets as well (O'Malia et al. (2010)). The search for diffuse band carriers in other environments is still very limited in scope mostly due to observational difficulties. For example, it is not known if diffuse band carriers could be present in HiI regions, dense molecular clouds, planetary nebulae, supernova remnants, proto-planetary disks, or other environments in which PAHs are observed.

How can this lack be explained? The absence of diffuse band carriers in other environments could be due to the fact that the "parent" carriers get modified (such as ionised or deprotenated or hydrogenated) only sufficiently in the harsh environment of the diffuse 
Table 4. Recent proposals for DIB carriers.

\begin{tabular}{|c|c|c|c|}
\hline Candidate carrier & | DIBs (Å) & Reference & Remark \\
\hline $\mathrm{C}_{60}^{+}$ & |9577, 9632 & $\begin{array}{l}\text { Foing \& Ehrenfreund (1994), } \\
\text { Foing \& Ehrenfreund (1997) }\end{array}$ & $\begin{array}{l}\text { No gas-phase spectra to verify peak } \\
\text { positions }\end{array}$ \\
\hline Carbon nano-tubes & - & Zhou et al. $(2006)$ & Calculated transitions. Non-specific. \\
\hline $\mathrm{CH}_{2} \mathrm{CN}^{-}$ & 8037 & Cordiner \& Sarre (2007) & $\begin{array}{l}\text { Calculated. Match in position. } \\
\text { Needs lab data to confirm. }\end{array}$ \\
\hline Fullerenes & 4428 & Iglesias-Groth et al. (2008) & $\begin{array}{l}\text { Theoretical calculation. Inaccurate } \\
\text { peak position. }\end{array}$ \\
\hline Large "floppy" molecule" & $\begin{array}{l}6863 \\
6844 \\
6203,5545\end{array}$ & Duley \& Kuzmin (2010) & $\begin{array}{l}\text { Origin bands of vibronic progres- } \\
\text { sion. Non-specific carrier. }\end{array}$ \\
\hline Proflavine (neutral \& ions) & $\begin{array}{l}4066, \\
4363 \\
4176,4259\end{array}$ & $\begin{array}{l}\text { Bonaca } \\
(2010)\end{array} \quad \& \quad$ Bilalbegović & $\begin{array}{l}\text { Calculated. Large offset in peak po- } \\
\text { sition. }\end{array}$ \\
\hline $\mathrm{HC}_{4} \mathrm{H}^{+}$ & 5068.8 & Krełowski et al. (2010) & Match in position, not of profile. \\
\hline Acetylene plasma & 5449 & Linnartz et al. (2010) & $\begin{array}{l}\text { Match in peak position and profile } \\
\text { shape. Non-specific carrier. }\end{array}$ \\
\hline l- $\mathrm{C}_{3} \mathrm{H}_{2}$ & $\begin{array}{l}4881 \\
5449 \\
6150-6330\end{array}$ & $\begin{array}{l}\text { Maier et al. (2011), } \\
\text { Oka \& McCall, }(2011)\end{array}$ & $\begin{array}{l}\text { Peak position and width match for } \\
5449 \text {, less for } 4887 \text { band. No convinc- } \\
\text { ing match for } 6150-6330 \text { bands. }\end{array}$ \\
\hline
\end{tabular}

Notes: The recent tentative detection of naphthalene and anthracene Iglesias-Groth et al. (2008), Iglesias-Groth et al. (2008) refuted by Searles et al. (2011), Galazutdinov et al. (2011) is not included as it did not pertain to an existing DIB.

ISM such that the "new" species can give rise to optical absorption bands. Also, it is possible that the carriers are destroyed in PDRs/HII regions; carriers deplete from the gas phase into the ice-mantle of dust grains in cold, dense clouds.

\section{Quest for the identity of the DIB carriers}

As mentioned above the debate on the gas or dust origin of the diffuse band carriers was started already in the 1930s. Free flying molecules were slightly favoured in those early days. In the 40s and 50s dust grains were in vogue mainly due to many new developments in the study of dust particles and the idea that large molecules could not be formed / survive in space. With the detection of polyatomic molecules in space (via rotational line emission at radio frequencies) the question presented itself again if the DIBs could perhaps be gas phase molecules. The line widths indicated that these molecules had to be significantly larger than the known di-atomic species (Platt (1956), Danks \& Lambert (1976)).

\section{PAH-DIB hypothesis}

With the discovery of infrared emission bands and their subsequent assignment to aromatics and fullerenes (Alamandola et al. (1985), Puget et al. (1985)), it became apparent that very large molecules are abundant in space. The link between PAHs and DIBs was quickly made (Crawford et al. (1985), Leger \& d'Hendecourt (1985), Van der Zwet \& Allamandola (1985)) as the optical properties (electronic absorption spectra) were known from laboratory studies (e.g. Birks (1970)). Apart from having (electronic) transitions in the UV, optical and near-infrared, PAHs are also very resistant to UV radiation. Thus the abundance of PAHs in space, their optical absorption spectrum and the presence of substructure in DIB profiles consistent with rotational profiles of intermediate-to-large PAHs all give strong support for the PAH-DIB hypothesis (Salama et al. (1999), Cox (2011)). A potential problem with the PAH-DIB (and PAH-UIR band) hypothesis is the lack of interstellar bands in both the UV (Snow \& Destree (2011), Snow et al. (2011)) and near-infrared part of the astronomical spectrum. 
Theoretical calculation of the main electronic transitions of small to intermediate PAHs (10 to 42 carbon atoms) show that the neutrals absorb in the UV, clustering between 2500-4000 A. Cations and anions absorb in the visible/near-infrared (4000-14000 ^). For both the neutrals and ions the transitions are seen to shift redwards with increasing size of the PAH (Ruiterkamp et al. (2005)). Also, other carbon-based molecules, such linear chains and fullerenes, absorb in the optical. The latter have been detected in space via their infrared emission (Cami et al. (2010)). The addition of side groups to PAHs (such as a methyl group or a small carbon chain) or the nitrogen or isotopic substitution and (de)hydrogenation of PAHs all alter the specific electronic configuration and thus the corresponding absorption spectrum (see e.g. Hammonds et al. (2011), Hammonds et al. (2011) for the effect of (de)protonation). Not only PAHs, but also their derivatives, and related structures such as fullerenes, carbon chains and rings are now also to be considered inclusive in the term "PAH" when discussing the PAH-DIB hypothesis.

\section{DIB carrier candidates}

With the growing number of claims for DIB carriers idependently verifiable criteria are probably warranted. In order of importance (high to low) the following criteria could be considered to be necessary for an identification of a DIB carrier (see e.g. also Galazutdinov et al. 2011):

(1) An exact match between the profile (position and shape) of the laboratory gasphase low-temperature spectra and the DIB profile is required.

(2) All similarly strong transitions (preferably at least two) detected in the laboratory should also be confirmed in the astronomical spectra.

(3) Detection in at least two separate lines-of-sight

For the identification of a specific PAH (i.e. not related to a known DIB) the criteria for identification are equally strict but slightly different as the feature is potentially new. We add the following conditions, that ensure the new feature is real and of interstellar origin:

(4) Its interstellar nature needs to be confirmed; e.g. to be stationary in a spectroscopic binary and/or its strength shows a positive trend with reddening

(5) The features should be absent toward unreddened lines-of-sight.

(6) Independent detection with different instruments to avoid instrumental artefacts.

In order to solve the DIB problem, one needs to find a specific PAH or related structure (or class of molecules) that has a limited set of very strong absorption bands (and possibly a larger number of weaker bands) and is either more abundant in space or is a more efficient absorber than most other large carbonaceous molecules. However, none of the candidate PAHs, fullerenes, or chains, thus far measured in the laboratory has been found to match any of the DIBs (Salama et al. (2008)). This is partly due to the fact that most spectra have been obtained in matrix isolation and only very few species have been measured at cold temperatures in the gas phase. Recent studies have focused on obtaining laboratory spectroscopic data of low temperature gas-phase neutral PAHs to compare with astronomical spectra of diffuse and translucent clouds (e.g. Pino et al. (2011), Salama et al. (2011), Salama et al. (2011), Gredel et al. (2011), Dietsche et al. (2011)). Ten different PAHs (containing 2 to 13 benzene rings / 11 to 42 carbon atoms) were searched for but none were detected in the ISM by both Salama et al. (2011) and Gredel et al. (2011) independently. In some cases there is a decent match in shape and position for a single laboratory band with a DIB (e.g. Pentacene and 5362.8 A DIB), however the shift and widths are still too different and other strong bands seen in the laboratory spectra are not observed in the ISM (Salama et al. (2011)). Typical column densities limits range from $10^{11}$ to $10^{14} \mathrm{~cm}^{-2}$ (Salama et al. (2011), Gredel et al. (2011)). Laboratory experiments on PAH cations and anions - whose spectra are expected to give 
more absorption bands in the red - are challenging with current experimental setups. Tentative detections of broad bands corresponding to naphthalene cation (Iglesias-Groth et al. (2008)) and anthracene cation (Iglesias-Groth et al. (2010)) were reported for one peculiar line-of-sight, but could not be confirmed in other sightlines (Searles et al. (2011), Galazutdinov et al. (2011)).

Table 4 gives a overview of recent DIB carrier identifications reported in literature, none of which is confirmed or without question. The tentative assignment of $C_{60}^{+}$to the 9577 and 9632 bands is still debated (Jenniskens et al. (1997), Galazutdinov et al. (2000), Ehrenfreund et al. (2006)). Unfortunately, the strong telluric absorption in this spectral range hampers detection of additional - weaker - bands from $C_{60}^{+}$. Results for calculated transitions or inferred vibronic progressions of large molecules are promising but await further study and confirmation from lab experiments. This is also the case for the good comparison between a hydrocarbon plasma band and the 5449 DIB (Linnartz et al. 2010). Mismatches, however small, for $\mathrm{CH}_{2} \mathrm{CN}^{-}$(Krelowski et al. 2010) and $\mathrm{HC}_{4} \mathrm{H}^{+}$ (Cordiner \& Sarre 2007) do not allow for a positive carrier identification. The recent assignment of $1-\mathrm{C}_{3} \mathrm{H}_{2}$ to several DIBs (Maier et al. (2011), Raghunandan et al. (2011)) has been challenged by others, revealing inconsistencies between the observations and laboratory data (Oka \& McCall, (2011), Krelowski (this volume)).

\section{Conclusions}

At present, the most promising candidate DIB carriers are large carbon molecules - in their various guises - that need to be stable, UV resistant, but sensitive to local cloud conditions (i.e. ISRF). However, alternative candidates cannot be dismissed a priori. Studying the presence and behaviour of DIBs in our Galaxy, Local Group and far beyond will hopefully lead to further insights into the formation and destruction mechanisms that govern the DIB carrier abundance. On the other hand, there exists now also an opportunity to use DIBs as a tracer of the physical and chemical conditions of the ISM in distant galaxies.

Spectroscopic signatures of PAHs and related structures are predicted to arise in both the UV (mainly neutrals) and near-infrared (predominantly large anions and cations). The observation of these transitions is crucial to both their assignment to the infrared emission bands as well as their link to the DIBs.

The apparent absence of DIB carriers - as opposed to PAHs - in various other environments such as circumstellar envelopes of evolved stars, comets, H II regions, etc., needs to be understood and can possible lead to new understandings leading to their identity.

Laboratory astrophysics is continuously developing new techniques to measure the optical spectra of complex molecules under interstellar space conditions. It is now increasingly feasible to make direct comparisons between laboratory data and astronomical observations. This leads to questions about the criteria that need to be met in order to firmly establish - beyond doubt - any identification. A possible set of criteria - open to discussion - is provided.

\section{Acknowledgement}

My thanks go to the SOC and LOC for organising an enjoyable and interesting meeting as well as for financial support. I thank Dennis Vos for preparing Figure 2. I also acknowledge the many fruitful and lively discussions I have had with my collaborators over the years and during this symposium in particular. 


\section{References}

Adamson, A. J., Whittet, D. C. B., \& Duley, W. W. 1990, MNRAS, 243, 400

Allamandola, L. J., Tielens, A. G. G. M., \& Barker, J. R. 1985, ApJ Suppl., 71, 733

Birks, J. B. 1970, Photophysics of Aromatic Molecules, John Wiley and Sons, Ltd., London

Bless, R. C. \& Savage, B. D. 1972, ApJ, 171, 293

Bonaca, A. \& Bilalbegović, G. 2010, Chemical Physics (Lett.), 493, 33

Butchart, I., McFadzean, A., Whittet, D., Geballe, T., \& Greenberg, J. 1986, A\& A, 154, 5

Cami, J., Salama, F., Jiménez-Vicente, J., Galazutdinov, G., \& Krełowski, J. 2004, ApJ, 611, 113

Cami, J., Bernard-Salas, J., Peeters, E., \& Malek, S. E. 2010, Science, 329, 1180

Cami, J., Bernard-Salas, J., Peeters, E., \& Malek, S. E. 2011, IAU Symp. 280, this volume

Cernicharo, J., Goicoechea, J. R., \& Caux, E. 2000, ApJ (Lett.), 534, 199

Chiar, J. E. \& Pendleton, Y. J. 2008, IAU Symposium 251, Eds. S. Kwok \& S. Sandford, 35

Cordiner, M. A., Cox, N. L. J., Trundle, C., Evans, C. J., Hunter, I., Przybilla, N., Bresolin, F., \& Salama, F. 2008, A\&A, 480, 13

Cordiner, M. A., Smith, K. T., Cox, N. L. J., Evans, C. J., Hunter, I., Przybilla, N., Bresolin, F., \& Sarre, P. J. 2008, A\& $A, 492,5$

Cordiner, M. A., Cox, N. L. J., Evans, C. J., Trundle, C., Smith, K. T., Sarre, P. J., \& Gordon, K. D. 2011, ApJ, 726, 39

Cordiner, M., Cox, N., Smith, K., Evans, C., Trundle, C., Gordon, K., \& Sarre, P. 2011, IAU Symp. 280, Poster 2.21

Cordiner, M. A. \& Sarre, P. J. 2007, AE $A$, 472, 537

Cox, N. 2011, in: C Joblin \& A. G. G. M. Tielens (eds.), PAHs and the Universe, EAS, 46, 349

Cox, N. L. J.., Boudin, N., Foing, B. H., Schnerr, R. S., Kaper, L., Neiner, C., Henrichs, H., Donati, J.-F., \& Ehrenfreund, P. 2007, A\&A A, 464, 899

Cox, N. L. J. \& Cordiner, M. A. 2011, IAU Symposium 251, 237

Cox, N. L. J., Kaper, L., Foing, B. H., \& Ehrenfreund, P. 2005, A\&A, 438, 187

Cox, N. L. J., Cordiner, M. A., Cami, J., Foing, B. H., Sarre, P. J., Kaper, L., \& Ehrenfreund, P. 2006, $A \mathscr{E} A, 447,991$

Cox, N. L. J., Cordiner, M. A., Ehrenfreund, P., Kaper, L., Sarre, P. J., Foing, B. H., Spaans, M., Cami, J., Sofia, U. J., Clayton, G. C., Gordon, K. D., \& Salama, F. 2007, A\&AA, 470, 941

Cox, N., Ehrenfreund, P., Foing, B., d'Hendecourt, L., Salama, F., \& Sarre, P. 2011, A\&A, 531, A25

Cox, N. L. J. \& Patat, F. 2008, A\& A, 485, 9

Cox, N. L. J. \& Spaans, M. 2006, A\& $A, 451,973$

Crawford, M. K., Tielens, A. G. G. M., \& Allamandola, L. J. 1985, ApJ, 293, 45

Danks. A. C., Lambert, D. L. 1976, MNRAS, 174, 571

Destree, J. D., Snow, T. P., \& Eriksson, K. 2007, ApJ, 664, 909

Dietsche, R., Chakrabarty, S., Rice, C., Mazzotti, F., \& Maier, J. 2011, IAU Symp. 280, Poster 3.25

Draine, B. 2011, in: C Joblin \& A. G. G. M. Tielens (eds.), PAHs and the Universe, EAS, 46, 100

Duley, W., Kuzmin 2010, ApJ (Lett.), 712, 165

Ehrenfreund, P., Cami, J., Jiménez-Vicente, J., Foing, B. H., Kaper, L., Van der Meer, A., Cox, N., d'Hendecourt, L., Maier, J. P., Salama, F., Sarre, P. J., Snow, T. P., \& Sonnentrucker, P., 2002, ApJ (Lett.), 576, 117

Ehrenfruend, P., Cox, N., \& Foing, B. 2006, in "Natural Fullerenes and Related Structures of Elemental Carbon", Development in Fullerene Science, vol. 6, Rietmijer, F. (Ed.), 58

Ferrière, K. M. 2001, Reviews of Modern Physics, 73, 103

Foing, B. H. \& Ehrenfreund, P. 1994, Nature, 369, 296

Ehrenfreund, P. \& Foing, B. H. 1996, A\&A (Lett.), 307, 25

Foing, B. H. \& Ehrenfreund, P. 1997, A\&A (Lett.), 317, 59

Friedman, S. D., York, D. G., McCall, B. J., Dahlstrom, J., Sonnentrucker, P., Welty, D. E., Drosback, M. M., Hobbs, L. M., Rachford, B. L., \& Snow, T. P. 2011, ApJ, 727, 33 
Galazutdinov, G., Krelowski, J., Musaev, F. A., Ehrenfreund, P., \& Foing, B. H. 2000, MNRAS, 317,750

Galazutdinov, G., Musaev, F. A., Bondar, A. V., \& Krelowski, J. 2003, MNRAS, 345, 365

Galazutdinov, G., Lee, B-C., Song, I-O., Kazmierczak, M., \& Krełowski, J. 2011, MNRAS, 412, 1259

Gredel, R., Carpentier, Y., Rouille, G., Steglich, M., Huisken, F., \& Henning, Th. 2011, A\&̊A, 530, A26

Hammonds, M., Candian, A., \& Sarre, P. 2011, IAU Symp. 280, Poster 1.34

Hammonds, M., Pathak, A., Candian, A., \& Sarre, P. 2011, in: C Joblin \& A. G. G. M. Tielens (eds.), PAHs and the Universe, EAS, 46, 373

Heckman, T. \& Lehnert, 2000, ApJ, 537, 690

Heger, M. 1922, Lick Observatory Bulletin, 10, 141

Herbig, G. H. 1995, ARAESA, 33, 19

Hobbs, L. M., York, D. G., Snow, T. P., Oka, T., Thorburn, J. A., Bishof, M., Friedman, S. D., McCall, B. J., Rachford, B., Sonnentrucker, P., \& Welty, D. E. 2008, ApJ, 680, 1256

Hobbs, L. M., York, D. G., Thorburn, J. A., Snow, T. P., Bishof, M., Friedman, S. D., McCall, B. J., Oka, T., Rachford, B., Sonnentrucker, P., \& Welty, D. E. 2009, ApJ, 705, 32

Iglesias-Groth, S. 2004, ApJ (Lett.), 608, 37

Iglesias-Groth, S. 2007, ApJ (Lett.), 661, 167

Iglesias-Groth, S., Manchado, A., García-Hernández, D. A., González Hernández, J. I., \& Lambert, D. L. 2008, ApJ, 685, 55

Iglesias-Groth, S., Manchado, A., Rebolo, R., Gonzalez Hernandez, J. I., Garcia-Hernandez, D. A., \& Lambert, D. L. 2010, MNRAS, 407, 2157

Jenniskens, P. \& Désert, F.-X. 1994, A\&̈A Suppl., 106, 39

Jenniskens, P., Mulas, G., Porceddu, I., \& benvenuti, P. 1997, A\&A, 327, 337

Joblin, C., D'Hendecourt, L., Leger, A., \& Maillard, J. P. 1990, Nature, 346, 729

Junkkarinen, V. T., Cohen, R. D., Beaver, E. A., Burbidge, E. M., Lyons, R. W., \& Madejski, G. 2004, ApJ, 614, 658

Kerr, T., Hibbins, R., Miles, J., Fossey, S., Somerville, W., \& Sarre, P. 1996, MNRAS, 283, 105

Krełowski, J. \& Sneden, C. 1994, ASPC, 58, 12

Krełowski, J. \& Westerlund, B. E. 1988, A\& A, 190, 339

Krełowski, J., Ehrenfreund, P., \& Foing, B. H., et al. 1999, A\&A A, 347, 235

Krełowski, J., Beletsky, Y., Galazutdinov, G. A., Kołos, R., Gronowski, M., \& LoCurto, G. 2010, ApJ, 714, 64

Krełowski, J. \& Kazmierczak, M. 2011, IAU Symp. 280, Poster 2.50

Kwok, S. \& Sandford, S. 2008, Eds. IAU Symposium 251

Leger, A. \& D'Hendecourt, L. 1985, A\&̊A, 146, 81

Leger, A., D'Hendecourt, L., Verstraete, L., \& Schmidt, W. 1988, A\&A, 203, 145

Le Page, V., Snow, T. P., \& Bierbaum, V. M. 2003, ApJ, 584, 316

Linnartz, H., Wehres, N., van Winckel, H., Walker, G. A. H., Bohlender, D. A., Tielens, A. G. G. M., Motylewski, T., \& Maier, J. P. 2010, A\& A, 511, 3

Luna, R., Cox, N. L. J., Satorre, M. A., García Hernéz, D. A., Suárez, O., \& García Lario, P. 2008, A\&A, 480, 133

Maier, J. P., Lakin, N. M., Walker, G. A. H., \& Bohlender, D. A. 2001, ApJ, 553, 267

Maier, J. P., Walker, G. A. H., Bohlender, D. A., Mazzotti, F. J., Raghunandan, R., Fulara, J., Garkusha, I., \& Nagy, A. 2011, ApJ, 726, 41

Martin, P. G. \& Angel, J. R. P. 1974, ApJ, 188, 517

Mauron, N. \& Kendall, T. R., 2004, A\& A, 428, 535

McCall, B. J., Drosback, M. M., Thorburn, J. A., York, D. G., Friedman, S. D., Hobbs, L. M., Rachford, B. L., Snow, T. P., Sonnentrucker, P., \& Welty, D. E. 2010, ApJ, 708, 1628

McFadzean, A. D., Whittet, D. C. B., Bode, M. F., Adamson, A. J., \& Longmore, A. J. 1989, MNRAS, 241, 873

Megier, A., Krełowski, J., \& Weselak, T. 2005, MNRAS, 358, 563

Mennella, V., Colangeli, L., Bussoletti, E., Palumbo, P., \& Rotundi, A. 1998, ApJ (Lett.), 507, 177 
Merrill, P. W. \& Wilson, O. C. 1938, ApJ, 87, 9

Mookerjea, B., Giesen, T., Stutzki, J., Cernicharo, J., Goicoechea, J. R., \& Black, J. H. 2011, IAU Symp. 280, this volume

Mulas, G., Malloci, G., Joblin, C., \& Cecchi-Pestellini, C. 2011, in: C Joblin \& A. G. G. M. Tielens (eds.), PAHs and the Universe, EAS, 46, 327

Munari, U., Tomasella, L., Fiorucci, M., Bienaymé, O., Binney, J., Bland-Hawthorn, J., Boeche, C., Campbell, R., Freeman, K. C., Gibson, B., Gilmore, G., Grebel, E. K., Helmi, A., Navarro, J. F., Parker, Q. A., Seabroke, G. M., Siebert, A., Siviero, A., Steinmetz, M., Watson, F. G., Williams, M., Wyse, R. F. G., \& Zwitter, T. 2008, A\& A, 488, 969

Oka, T. \& McCall, B. J. 2011, Science, 331, 293

O'Malia, K. K. J.; Thorburn, J. A.; Hammergren, M.; Snow, T. P.; Dembicky, J.; Hobbs, L. M.; York, D. G. 2010, ApJ, 708, 785

Peeters, E. 2011, in: C Joblin \& A. G. G. M. Tielens (eds.), PAHs and the Universe, EAS, 46, 13

Peeters, E. 2011, IAU Symp. 280, this volume

Pendleton, Y. J., Sandford, S. A., Allamandola, L. J., Tielens, A. G. G. M., \& Sellgren, K. 1994, ApJ, 437, 683

Pino, T., Carpentier Y., Feraud. G., Friha, H., Kokkin, D. L., Troy, T. P., Chalyavi, N., Brechignac, Ph., \& Schmidt, T. W. 2011, in: C Joblin \& A. G. G. M. Tielens (eds.), PAHs and the Universe, EAS, 46, 355

Platt, J. R., 1956, ApJ, 123, 486

Puget, J. L., Leger, A., \& Boulanger, F. 1985, A\&A A, 142, 19

Raghunandan, R., Maier, J. P., Walker, G. A. H., Bohlender, D. A., Mazzotti, F. J., Fulara, J., Garkusha, I., \& Nagy, A. 2011, IAU Symp. 280, this volume

Rawlings, M., Adamson, A., McCall, B., \& Kerr, T. 2011, IAU Symp. 280, Poster 2.82

Roueff, E., Felenbok, P., Black, J. H., \& Gry, C. 2002, A\&A, 384, 629

Ruiterkamp, R., Cox, N.L.J., Spaans, M., Kaper, L., Foing, B.H., Salama, F., Ehrenfreund, P. $A \& A, 432,515$

Salama, F., Galazutdinov, G., Krełowski, J., Allamandola, L., \& Musaev, F. 1999, ApJ, 526, 265

Salama, F. 2008, IAU Symposium 251, Eds. S. Kwok \& S. Sandford, 357

Salama, F., Galazutdinov, G., Krelowski, J., Biennier, L., Beletsky, Y., \& Song, I. O. 2011, ApJ, 728,154

Salama, F., Galazutdinov, G., Krelowski, J., Biennier, L., Beletsky, Y., \& Song, I. O. 2011, IAU Symp. 280, Poster 2.88

Sandford, S. A., Pendleton, Y. J., \& Allamandola, L. J., 1995 ApJ, 440, 697

Sarre, P. J., Miles, J. R., Kerr, T. H., Hibbins, R. E., Fossey, S. J., \& Somerville, W. B. 1995, MNRAS, 277, 41

Sarre, P. J. 2006, Journal of Molecular Spectroscopy, 238, 1

Searles, J., Destree, J., Snow, T., Salama, F., York, D., \& Dahlstrom, J. 2011, ApJ, 732, 50

Snow, T. P. \& Destree, J. D. 2011, in: C Joblin \& A. G. G. M. Tielens (eds.), PAHs and the Universe, EAS, 46, 341

Snow, T. P. \& McCall, B. J. 2008, ARA\&A, 44, 367

Snow, T. P., Burgh, E. B., \& Destree, J. P. 2011, IAU Symp. 280, Poster 1.83

Sollerman, J., Cox, N., Mattila, S., Ehrenfreund, P., Kaper, L., Leibundgut, B., \& Lundqvist, P. 2005, $A \mathscr{E} A, 429,559$

Stecher, T. P. 1965, ApJ, 142, 1683

Thöne, C. C., Michałowski, M. J., Leloudas, G., Cox, N. L. J., Fynbo, J. P. U., Sollerman, J., Hjorth, J., \& Vreeswijk, P. M. 2009, ApJ, 698, 1307

Thorburn, J. A., Hobbs, L. M., McCall, B. J., Oka, T., Welty, D. E., Friedman, S. D., Snow, T. P., Sonnentrucker, P., \& York, D. G. 2003, ApJ, 484, 339

Tielens, A. 2008, ARA\&A, 46, 289

Tielens, A. 2011, in: C Joblin \& A. G. G. M. Tielens (eds.), PAHs and the Universe, EAS, 46, 3

Tuairisg, S.Ó., Cami, J., Foing, B. H., Sonnentrucker, P., \& Ehrenfreund, P. 2000, A $\mathscr{J} A$ Suppl., 142,225

Van der Zwet, G. P. \& Allamandola, L. J. 1985, Aध A, 146, 76 
Vos, D. A. I., Cox., N. L. J., Kaper, L., Spaans, M., \& Ehrenfreund, P. 2011, in preparation Welty, D., Federman, S., Gredel, R., Thorburn, J., \& Lambert, D. 2006, ApJ Suppl., 165, 138 Wallerstein, G., Sandstrom, K., \& Gredel, R. 2007, PASP, 119, 1268

Xiang, F. Y., Li, A., \& Zhong, J. X. 2011, ApJ, 733, 91

York, B. A., Ellison, S. L., Lawton, B., Churchill, C. W., Snow, T. P., Johnson, R. A., \& Ryan, S. G., 2006, 2006, 647, 29

Zhou, Z., Sfeir, M., Zhang, L., Hybertsen, M., Steigerwald, M., \& Brus, L. 2006, ApJ, 638, 105

\section{Discussion}

JACEK KRELOWSKI: According to our data the reported correlations between the 8621 DIB strength and reddening are too optimistic.

NICK Cox: This DIB is difficult to measure. It is relatively broad making the continuum placement more insecure. Also blending with stellar lines can be an issue, in particular in lower-resolution spectra of later spectral type stars used by Munari et al. In addition, it appears that also the uncertainties in $\mathrm{E}(\mathrm{B}-\mathrm{V})$ are now becoming more important as measurements of DIB strengths are increasingly more accurate. New surveys with accurate measurements of this diffuse band and extinction are needed to confirm these results.

Gwendolyn Meeus: You said that DIBs are not present in circumstellar envelope of evolved stars. What is the situation for the circumstellar environment of young stars?

NICK Cox: Currently, DIB carriers are not yet - conclusively - detected in environments other than the ISM. Studies have been performed, for example, for evolved stars, Herbig Ae/Be stars, and comets (see Sect. 6 of this chapter).

MARK RAWLINGS: Have there been any attempts to measure solid state hydrocarbon features (e.g. $3.4 \mu \mathrm{m}$ feature) towards the $8620.4 \AA$ sightlines.

NICK Cox: Most sightlines for which the $8621 \AA$ DIB has been measured are not heavily reddened $(\mathrm{E}(\mathrm{B}-\mathrm{V})<2 \mathrm{mag})$. It would be interesting to explore the connection between the $3.4 \mu \mathrm{m}$ feature (believed to be due to solid-phase aliphatic hydrocarbons) and the $8621 \AA$ DIB (with an apparent strong relation to dust grains) in sufficiently reddened sightlines. 